\title{
Pain Processing Is Faster than Tactile Processing in the Human Brain
}

\author{
Markus Ploner, Joachim Gross, Lars Timmermann, and Alfons Schnitzler \\ Department of Neurology, Heinrich-Heine-University, D-40225 Düsseldorf, Germany
}

Pain signals threat and drives the individual into a behavioral response that significantly depends on a short stimulus-response latency. Paradoxically, the peripheral and spinal conduction velocities of pain are much slower than of tactile information. However, cerebral processing times and reaction times of touch and pain have not yet been fully assessed. Here we show that reaction times to selective nociceptive cutaneous laser stimuli are substantially faster than expected from the peripheral conduction velocities. Furthermore, by using magnetoencephalography, we found that latencies between earliest stimulus-evoked cortical responses and reaction times are $\sim 60$ $\mathrm{ms}$ shorter for nociceptive than for tactile stimuli. These findings reveal that cerebral processing of pain is substantially faster than processing of tactile information and relatively compensates for the slow peripheral and spinal conduction velocities of pain. Our observation shows how the cerebral organization of pain processing enhances motor responses to potentially harmful stimuli and thereby subserves the particular behavioral demands of pain.

Key words: pain; nociception; touch; somatosensory cortices; reaction times; magnetoencephalography

\section{Introduction}

The physical integrity of the individual critically depends on the perception of pain. Central to the protective function of pain are fast and effective behavioral responses to potentially harmful stimuli. Accordingly, pain drives the individual into a motor response aimed at achieving safety and promoting recovery.

Research during the past decades revealed that the experience of pain is subserved by physiologically specialized neural pathways termed the nociceptive system (Craig, 2003; Willis and Westlund, 2004). The peripheral part of the nociceptive system consists of thinly myelinated $\mathrm{A} \delta$ fibers and unmyelinated $\mathrm{C}$ fibers, although some nociceptive $A \beta$ fibers have also been described (Willis and Westlund, 2004; Meyer et al., 2006). In primates, the peripheral pain pathways and the major spinal pain pathway (Ferrington et al., 1987; Kakigi and Shibasaki, 1991; Dostrovsky and Craig, 2006) mainly show paradoxically slow conduction velocities of $10-20 \mathrm{~m} / \mathrm{s}$, which may provide a slow but particular safe pathway of nociceptive information to the brain. However, because of the low peripheral and spinal conduction velocities, pain-related information from the hand arrives at the human brain not earlier than $\sim 100 \mathrm{~ms}$ after stimulus application.

At the cortical level, an extended network is associated with pain processing, which includes primary (S1) and secondary (S2) somatosensory cortices, as well as insular, anterior cingulate, and prefrontal cortices (Bushnell and Apkarian, 2006). These areas

\footnotetext{
Received Aug. 7, 2006; revised Sept. 8, 2006; accepted Sept. 10, 2006.

This work was supported by the German Ministry of Education and Science (Bundesministerium für Bildung und Forschung Grant 01GW0533). We thank C. Ploner, B. Hommel and L. Plaghki for helpful comments on this manuscript.

Correspondence should be addressed to Dr. Markus Ploner, Department of Neurology, Heinrich-Heine-University, Moorenstrasse 5, 40225 Düsseldorf, Germany. E-mail: ploner@neurologie.uni-duesseldorf.de. DOI:10.1523/JNEUROSCI.3398-06.2006

Copyright $\odot 2006$ Society for Neuroscience ～0270-6474/06/2610879-04\$15.00/0
}

participate in different sensory, cognitive, and affective processes and, thus, differentially contribute to the experience of pain. Time courses of pain-evoked activations (Ploner et al., 1999; Kanda et al., 2000; Ohara et al., 2004) indicate that parts of this network are organized in parallel, which contrasts to the rather serial processing of other somatosensory information (Kaas, 2004). However, within this cortical network, processing times of behavioral responses to pain have remained essentially unknown. To address this question, we recorded neuromagnetic brain responses during a reaction time experiment and directly compared latencies of cortical responses and reaction times to painful and tactile stimuli. We hypothesized that the cortical organization of pain processing may allow for particularly fast processing of pain-related information to effectively prevent harm. Our results reveal that latencies between earliest stimulus-evoked cortical responses and reaction times are $\sim 60 \mathrm{~ms}$ shorter for nociceptive than tactile stimuli. However, latencies between responses from S2 and reaction times did not differ between modalities, suggesting that the direct thalamic access of pain-related information to S2 may underlie the short central processing time of pain. These observations show how the cortical organization of pain processing relatively compensates for the slow peripheral and spinal conduction velocities of pain and thereby subserves the particular behavioral demands of pain.

\section{Materials and Methods}

Twelve healthy male subjects with a mean age of 30 years (range, 22-39 years) participated in the study. Informed consent was obtained from all subjects before participation. The study was approved by the local ethics committee and was conducted in conformity with the Declaration of Helsinki. Two subjects had to be excluded from the analysis because of an extremely low signal-to-noise ratio. Thus, analysis was based on 10 subjects.

Procedure. In a simple reaction time experiment, each of 60 painful and nonpainful stimuli was randomly applied to the right hand. Interstimulus interval was varied between 6 and $10 \mathrm{~s}$. Subjects were instructed to 


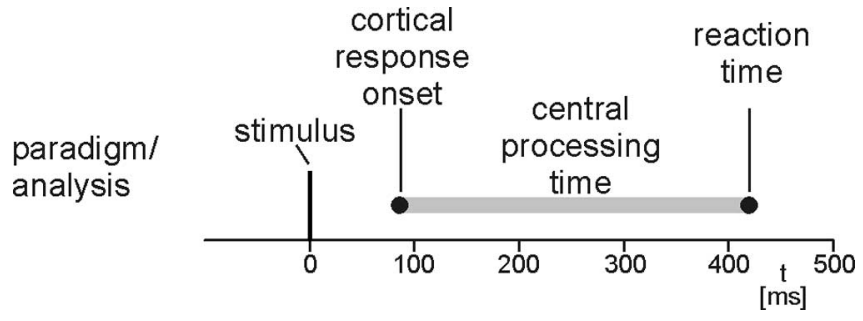

Figure 1. Paradigm and analysis. Stimuli were painful and tactile stimuli randomly applied to the right hand. Reaction times were button presses with the index finger of the left hand. Central processing time was defined as latency between earliest stimulus-evoked cortical responses and reaction times.

react as fast as possible to the stimuli by pressing a button with the index finger of the left hand. Before the experiment, a practice block of at least 20 stimuli was performed.

Stimulation. Painful stimuli were applied to the dorsum of the right hand by using cutaneous laser stimulation, which has been shown to activate selectively nociceptive $\mathrm{A} \delta$ and $\mathrm{C}$ afferents (Bromm and Treede, 1984). The laser device was a Tm:YAG laser (Carl Baasel Lasertechnik, Starnberg, Germany) with a wavelength of $2000 \mathrm{~nm}$, a pulse duration of $1 \mathrm{~ms}$, and a spot diameter of $6 \mathrm{~mm}$. The laser beam was led through an optical fiber from outside into the recording room. Stimulation site was slightly changed within an area of $4 \times 3 \mathrm{~cm}$ after each stimulus. Applied stimulus intensity was twice pain threshold intensity, which resulted in stimulus intensities between 550 and $700 \mathrm{~mJ}$, evoking moderately painful sensations. Mean pain rating on a numerical rating scale from 0 to 10 , with end points "no pain" and "worst possible pain", was 3.5.

Tactile stimuli were constant voltage electrical pulses of $0.3 \mathrm{~ms}$ duration delivered to the middle and end phalanx of the right index finger by using ring electrodes. Stimulus intensity was adjusted to twice detection threshold intensity, i.e., 12-16 V, thus inducing clear and consistent nonpainful sensations.

Recordings and analysis. Subjects were comfortably seated with eyes closed in a magnetically shielded room. Environmental noise was masked by white noise applied to both ears.

Reaction times to painful and tactile stimuli were calculated as latency between stimulus application and button press. Reaction times shorter than $100 \mathrm{~ms}$ or longer than $800 \mathrm{~ms}$ were discarded. Median reaction times were calculated, and outliers below or above 2 SDs of the median were discarded.

Cortical activity was recorded with a Neuromag- 122 whole-head neuromagnetometer (Elekta, Stockholm, Sweden) containing 122 planar SQUID gradiometers. Signals were digitized at $1020 \mathrm{~Hz}$, high-pass filtered at $1 \mathrm{~Hz}$, and low-pass filtered at $120 \mathrm{~Hz}$. Neuromagnetic activity was averaged time-locked to application of tactile and painful stimuli. Vertical electrooculograms were used to reject epochs contaminated with blink artifacts.

Global stimulus-evoked neuromagnetic activity was calculated as root mean square of the signals of all 122 sensors corrected to baseline. Onsets of global stimulus-evoked responses were defined as time points at which cortical activity exceeds 2 SDs of baseline activity from -100 to $-10 \mathrm{~ms}$. The latency difference between response onsets and reaction times, termed central processing time, was determined as a measure of the central processing demands of painful and tactile stimuli in the simple reaction time experiment (Fig. 1).

Additional analysis of somatosensory and pain-evoked fields was based on a spatiotemporal source model (Hämäläinen et al., 1993). Sources of evoked responses were modeled as equivalent current dipoles identified during clearly dipolar field patterns. Only sources accounting for $>85 \%$ of the local field variance were accepted. Source locations, orientations, and strengths were calculated within a realistic head model (boundary-element model) of each subject's head determined from the individual magnetic resonance images acquired on a $1.5 \mathrm{~T}$ Siemens $(\mathrm{Mu}-$ nich, Germany) Magnetom. Individual locations of responses were transformed to normalized Talairach space, and group mean Talairach coordinates were calculated. Time courses of activations were obtained from the spatiotemporal source model in which locations and orientations of sources were kept fixed and activation strengths were allowed to vary over time to provide the best fit for the recorded data. Onsets of local stimulus-evoked responses were defined as time points at which activation strength exceeds 2 SDs of baseline activity from -100 to $-10 \mathrm{~ms}$. Latencies between $\mathrm{S} 1$ and $\mathrm{S} 2$ responses to both stimuli and reaction times were calculated.

Reaction times and latencies of global and local stimulus-evoked cortical responses were compared by using Friedman's ANOVA and twotailed Wilcoxon's signed-rank tests.

Control experiment. Reaction times tend to shorten with an increase in stimulus intensity (Brebner and Welford, 1980). Thus, we verified whether an intensity increase of the electrical stimuli may yield a shortening of reaction times, which could explain our observation of an $60 \mathrm{~ms}$ difference in central processing time between modalities.

Six of the 10 subjects participated in the control experiment (mean age, 30 years; range, 22-36 years). In principle, the procedure of the control experiment was the same as in the main experiment. The only difference was that, instead of a laser and an electrical stimulus, two different intensities of electrical stimuli were applied to the right index finger. Stimuli were constant voltage electrical pulses of $0.3 \mathrm{~ms}$ duration delivered to the middle and end phalanx of the right index finger by using ring electrodes. Stimulus intensity was adjusted to twofold and fourfold detection threshold intensity, i.e., $16-20$ and 32-40 V, respectively. Twofold detection threshold intensity induced clear and consistent nonpainful sensations, whereas fourfold detection threshold intensity induced clearly unpleasant, slightly painful sensations.

\section{Results}

Figure 2 shows individual (left) and group mean (right) results. The analysis reveals that reaction times to painful stimuli were 26 ms later than reaction times to tactile stimulation $(387 \pm 20$ vs $361 \pm 25 \mathrm{~ms}$, mean $\pm \mathrm{SEM} ; p<0.05$, Wilcoxon's signed-rank test). Likewise, onsets of global stimulus-evoked cortical responses were later for painful than for tactile stimulation (128 \pm 6 vs $36 \pm 2 \mathrm{~ms} ; p<0.01)$. This delay of cortical and behavioral responses to pain is attributable to the lower conduction velocity of nociceptive peripheral and spinal pathways (10-20 m/s) compared with tactile pathways ( $\sim 50 \mathrm{~m} / \mathrm{s}$ ) (Vallbo et al., 1979; Ferrington et al., 1987; Kakigi and Shibasaki, 1991; Meyer et al., 2006).

Calculation of central processing time as the latency between arrival of nociceptive information at the cortex and reaction time reveals a difference between pain processing and tactile processing. Central processing time was on average $62 \pm 14 \mathrm{~ms}$ shorter for painful than for tactile stimuli $(271 \pm 19$ vs $333 \pm 25 \mathrm{~ms} ; p<$ $0.01)$. This difference in central processing time was observed in all subjects. The magnitude of the difference indicates that the cortical organization of the perception-action sequence in our simple reaction time experiment differs substantially between modalities.

To specify further the different central processing times of painful and tactile stimuli, we localized early cortical responses to both stimuli and calculated time courses for each area and modality. The analysis confirms generation of early cortical responses to painful (Kakigi et al., 2005) and tactile (Hari and Forss, 1999) stimuli in S1 and S2 (Fig. 3). However, the temporal activation pattern of S1 and S2 differs between modalities. Painful stimuli nearly simultaneously activate S1 and contralateral S2 (S1, $125 \pm 9 \mathrm{~ms}$; contralateral S2, $117 \pm 6 \mathrm{~ms}$; ipsilateral S2, $124 \pm 5 \mathrm{~ms})$, whereas tactile stimuli sequentially activate these areas (S1, $32 \pm 1 \mathrm{~ms}$; contralateral S2, $67 \pm 2 \mathrm{~ms}$; ipsilateral S2, $88 \pm 4 \mathrm{~ms}$ ), which is in good accordance with previous investigations (Ploner et al., 1999; Kanda et al., 2000; Ohara et al., 2004), The intermodal difference in the temporal activation pattern of 
individual

group
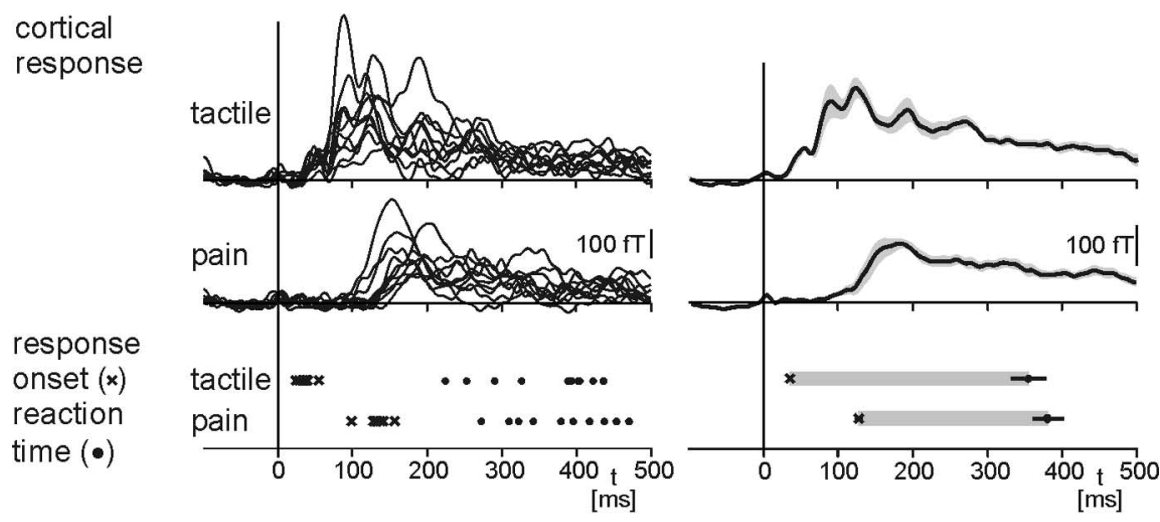

central
processing
time
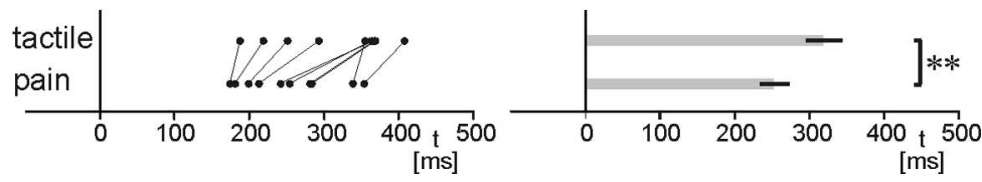

Figure 2. Cortical responses, reaction times, and central processing times to painful and tactile stimuli. Left and right show individual and group mean results, respectively. Cortical responses are global stimulus-evoked responses detected by all 122 magnetoencephalographic sensors over the whole head. Lines in the bottom left connect data points from the same individual. Shaded areas in the top right time course panels depict \pm SEM. Error bars refer to SEM. ${ }^{* *} p<0.01$, Wilcoxon's signed-rank test.
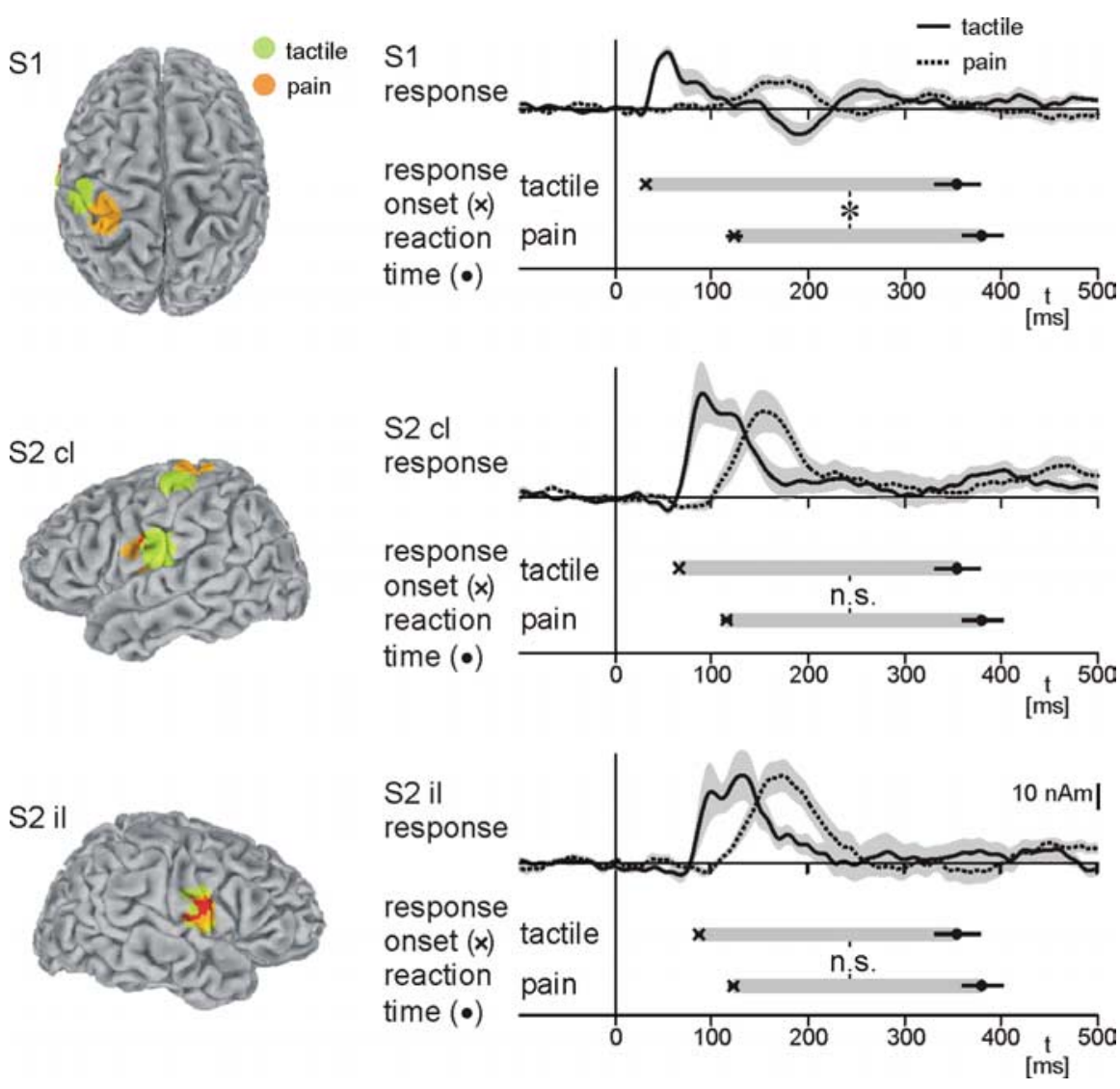

Figure 3. Local cortical responses, reaction times, and latencies between local cortical responses and reaction times. Locations of responses to painful and tactile stimulation were calculated as group mean normalized dipole locations. Mean coordinates of responses to painful stimuli are as follows: $S 1,-30,-34,65$; contralateral $S 2,-52,-4$, 18; ipsilateral $S 2,50,-8,15$. Mean coordinates of responses to tactile stimuli are as follows: $S 1,-46,-23,58$; contralateral $S 2,-56,-14,21$; ipsilateral S2 49, - 10, 20. The latency difference between earliest local responses (S1, contralateral S2, and ipsilateral S2) and reaction times represents the local equivalent of the central processing time of Figure 2, which refers to global cortical responses. Shaded areas in the time course panels depict \pm SEM. Error bars refer to SEM. cl, Contralateral; il, ipsilateral. ${ }^{*} p<0.05$, Wilcoxon's signed-rank test.
S1 and S2 most probably reflects a difference in the hierarchical organization of these areas. Pain-related information is conveyed in parallel from the thalamus directly to S1 and S2, whereas tactile information is transmitted serially from the thalamus to S1 and thence to S2 (Ploner et al., 1999; Kanda et al., 2000). Next, latencies between $\mathrm{S} 1$ and $\mathrm{S} 2$ responses to both stimuli and reaction times were calculated. Statistical analysis reveals a significant effect of modality (pain and tactile; $p<0.01$, Friedman's ANOVA) and area (S1, contralateral S2, and ipsilateral S2; $p<0.01$, Friedman's ANOVA) on latencies between local cortical responses and reaction times. Post hoc testing shows that latencies between $\mathrm{S} 1$ responses and reaction times differ significantly between modalities (pain, $274 \pm 18 \mathrm{~ms}$; tactile, $337 \pm$ $26 \mathrm{~ms} ; p<0.01$ ), whereas latencies between bilateral S2 responses and reaction times do not differ between modalities (contralateral S2: pain, $274 \pm 18 \mathrm{~ms}$; tactile, $302 \pm 26 \mathrm{~ms} ; p=0.22$; ipsilateral S2: pain, $275 \pm 19 \mathrm{~ms}$; tactile, $281 \pm 25 \mathrm{~ms}$; $p=0.76)$.

In the control experiment, reaction times to stronger and painful electrical stimuli were $15 \mathrm{~ms}$ shorter than reaction times to standard electrical stimuli (strong, $265 \pm 19 \mathrm{~ms}$; standard, $280 \pm 17$ $\mathrm{ms})$. Thus, an intensity difference between stimuli is unlikely to account for the $60 \mathrm{~ms}$ difference in central processing time between modalities.

\section{Discussion}

Here, we show that, in a simple reaction time experiment, cortical processing of pain is substantially faster than processing of tactile information. To the very best of our knowledge, no study has yet directly compared the central processing times of selective nociceptive and tactile stimuli. Reaction times of the present study are in good accordance with previous studies investigating reaction times to painful laser stimuli (Campbell and LaMotte, 1983; Pertovaara et al., 1984; Arendt-Nielsen and Bjerring, 1988). A single study directly compared reaction times to both stimuli (Pertovaara et al., 1984). In this study, reaction times to painful stimuli were $\sim 60$ ms longer than reaction times to tactile stimulation. Considering the longer receptor activation latency of their nociceptive stimulus, these results correspond well to our findings.

The delay of cortical responses and reaction times to painful stimuli compared with tactile stimuli is attributable to the low conduction velocity of nociceptive af- 
ferents. Peripheral (Meyer et al., 2006) and spinal (Ferrington et al., 1987; Kakigi and Shibasaki, 1991) nociceptive afferents conduct at $\sim 10-20 \mathrm{~m} / \mathrm{s}$, whereas conduction velocities of tactile afferents amount to $\sim 50 \mathrm{~m} / \mathrm{s}$ (Vallbo et al., 1979). Consequently, in intracranial and extracranial recordings, earliest responses to selective nociceptive stimuli from the hand are typically recorded at $\sim 120$ ms (Ploner et al., 1999; Kanda et al., 2000; Ohara et al., 2004; Kakigi et al., 2005). Only recently, S1 responses to painful stimuli at latencies of $\sim 90 \mathrm{~ms}$ have been reported (Inui et al., 2003), but even these latencies cannot explain our present observation of a $60 \mathrm{~ms}$ difference in central processing time between modalities. The functional significance of the slow peripheral and spinal conduction velocities of pain has remained unknown. However, the thinly myelinated nociceptive afferents are less vulnerable to mechanical, thermal, and chemical interference than the faster conducting tactile fibers. Thus, the peripheral conduction of pain may provide a slow but particular safe pathway of nociceptive information to the brain.

Assuming a common final motor pathway of behavioral responses to painful and tactile stimuli, our results indicate that the cortical organization of sensorimotor transformation differs between modalities. A difference in intensity is unlikely to account for the intermodal difference in sensorimotor transformation. The control experiment shows that even more intense tactile stimuli do not yield a shortening of processing times in the order of $60 \mathrm{~ms}$. Possibly, the difference in central processing time is caused by an intermodal difference in sensory pathways to the motor system. Anatomical data indicate three plausible candidates for conveying pain-related information to the motor system. These areas are the midcingulate cortex (Vogt et al., 2004), the posterior parietal cortex, and S2 (Kaas, 2004). Based on neither previous nor present data can we decide which area represents the crucial relay to the motor system. However, the crossmodally stable temporal relationship between $\mathrm{S} 2$ responses and reaction times would be well compatible with an important role of S2 for conveying somatosensory information to the motor system. Such a relay function of S2 is supported by anatomical (Krubitzer and Kaas, 1990; Disbrow et al., 2003), neurophysiological (Huttunen et al., 1996; Forss and Jousmaki, 1998), and functional imaging (Ledberg et al., 1995; Binkofski et al., 1999) evidence. Thus, in the present simple reaction time experiment, both tactile and pain-related information may be routed to the motor system via the S2 region. The parallel organization of pain processing with a direct thalamic access of pain-related information to S2 may then allow for the particular short central processing time of pain.

In conclusion, the present findings show that, in a simple reaction time experiment, cortical processing of pain is faster than processing of tactile information. The short cortical processing time of pain may be subserved by the parallel organization of somatosensory cortices in human pain processing, which relatively compensates for the slow peripheral and spinal conduction velocities of nociceptive pathways, enhances motor responses to potentially harmful stimuli, and thereby subserves the particular behavioral demands of pain.

\section{References}

Arendt-Nielsen L, Bjerring P (1988) Reaction times to painless and painful $\mathrm{CO} 2$ and argon laser stimulation. Eur J Appl Physiol Occup Physiol 58:266-273.

Binkofski F, Buccino G, Posse S, Seitz RJ, Rizzolatti G, Freund H (1999) A fronto-parietal circuit for object manipulation in man: evidence from an fMRI-study. Eur J Neurosci 11:3276-3286.
Brebner JMT, Welford AT (1980) Introduction: an historical background sketch. In: Reaction times (Welford AT, ed), pp 1-23. London: Academic. Bromm B, Treede RD (1984) Nerve fibre discharges, cerebral potentials and sensations induced by CO2 laser stimulation. Hum Neurobiol 3:33-40.

Bushnell MC, Apkarian AV (2006) Representation of pain in the brain. In: Wall and Melzack's textbook of pain (McMahon SB, Koltzenburg M, eds), pp 107-124. Philadelphia: Elsevier.

Campbell JN, LaMotte RH (1983) Latency to detection of first pain. Brain Res 266:203-208.

Craig AD (2003) Pain mechanisms: labeled lines versus convergence in central processing. Annu Rev Neurosci 26:1-30.

Disbrow E, Litinas E, Recanzone GH, Padberg J, Krubitzer L (2003) Cortical connections of the second somatosensory area and the parietal ventral area in macaque monkeys. J Comp Neurol 462:382-399.

Dostrovsky JO, Craig AD (2006) Ascending projection systems. In: Wall and Melzack's textbook of pain (McMahon SB, Koltzenburg M, eds), pp 187-203. Philadelphia: Elsevier.

Ferrington DG, Sorkin LS, Willis Jr WD (1987) Responses of spinothalamic tract cells in the superficial dorsal horn of the primate lumbar spinal cord. J Physiol (Lond) 388:681-703.

Forss N, Jousmaki V (1998) Sensorimotor integration in human primary and secondary somatosensory cortices. Brain Res 781:259-267.

Hämäläinen M, Hari R, Ilmoniemi RJ, Knuutila J, Lounasmaa OV (1993) Magnetoencephalography: theory, instrumentation, and applications to noninvasive studies of the working human brain. Rev Mod Phys 65:413-497.

Hari R, Forss N (1999) Magnetoencephalography in the study of human somatosensory cortical processing. Philos Trans R Soc Lond B Biol Sci 354:1145-1154.

Huttunen J, Wikstrom H, Korvenoja A, Seppalainen AM, Aronen H, Ilmoniemi RJ (1996) Significance of the second somatosensory cortex in sensorimotor integration: enhancement of sensory responses during finger movements. NeuroReport 7:1009-1012.

Inui K, Tran TD, Qiu Y, Wang X, Hoshiyama M, Kakigi R (2003) A comparative magnetoencephalographic study of cortical activations evoked by noxious and innocuous somatosensory stimulations. Neuroscience 120:235-248.

Kaas JH (2004) Somatosensory system. In: The human nervous system (Paxinos G, Mai JK, eds). Amsterdam: Elsevier.

Kakigi R, Shibasaki H (1991) Estimation of conduction velocity of the spinothalamic tract in man. Electroencephalogr Clin Neurophysiol 80:39-45.

Kakigi R, Inui K, Tamura Y (2005) Electrophysiological studies on human pain perception. Clin Neurophysiol 116:743-763.

Kanda M, Nagamine T, Ikeda A, Ohara S, Kunieda T, Fujiwara N, Yazawa S, Sawamoto N, Matsumoto R, Taki W, Shibasaki H (2000) Primary somatosensory cortex is actively involved in pain processing in human. Brain Res 853:282-289.

Krubitzer LA, Kaas JH (1990) The organization and connections of somatosensory cortex in marmosets. J Neurosci 10:952-974.

Ledberg A, O’Sullivan BT, Kinomura S, Roland PE (1995) Somatosensory activations of the parietal operculum of man. A PET study. Eur J Neurosci 7:1934-1941.

Meyer RA, Ringkamp M, Campbell JN, Raja SN (2006) Peripheral mechanisms of cutaneous nociception. In: Wall and Melzack's textbook of pain (McMahon SB, Koltzenburg M, eds), pp 3-34. Philadelphia: Elsevier.

Ohara S, Crone NE, Weiss N, Treede RD, Lenz FA (2004) Amplitudes of laser evoked potential recorded from primary somatosensory, parasylvian and medial frontal cortex are graded with stimulus intensity. Pain 110:318-328.

Pertovaara A, Reinikainen K, Hari R (1984) The activation of unmyelinated or myelinated afferent fibers by brief infrared laser pulses varies with skin type. Brain Res 307:341-343.

Ploner M, Schmitz F, Freund HJ, Schnitzler A (1999) Parallel activation of primary and secondary somatosensory cortices in human pain processing. J Neurophysiol 81:3100-3104.

Vallbo AB, Hagbarth KE, Torebjork HE, Wallin BG (1979) Somatosensory, proprioceptive, and sympathetic activity in human peripheral nerves. Physiol Rev 59:919-957.

Vogt BA, Hof PR, Vogt LJ (2004) Cingulate gyrus. In: The human nervous system (Paxinos G, Mai JK, eds), pp 915-949. Amsterdam: Elsevier.

Willis WD, Westlund KN (2004) Pain system. In: The human nervous system (Paxinos G, Mai JK, eds), pp 1125-1170. Amsterdam: Elsevier. 